\title{
Wer ist der ideale Kombinationspartner für Metformin?
}

_ Beim Typ-2-Diabetes gibt es nach Dr. Michael Jecht, Berlin, zwei Kernaspekte: die zu erwartende Zunahme der Prävalenz sowie das Einsparpotenzial durch Verbesserung der Adhärenz. So gehe man in den Vereinigten Staaten von Amerika von einer Zunahme der Typ2-Diabetes-Fälle von 22\% der Bevölkerung im Jahr 2011 auf 37\% im Jahr 2050 aus. Die Behandlungskosten würden von 120 Mrd. US\$ (30\% der Gesamtausgaben) auf 350 Mrd. US\$ (50\% der Gesamtausgaben) ansteigen. Eine Verbesserung der Adhärenz könne die Gesamtbehandlungskosten pro Patient jedoch um bis zu 250.000 US\$ reduzieren. Dies übersteige das Potenzial selbst einer nur um $25 \%$ effektiveren oralen Therapie, das 151.000 US\$/Patient kosten würde.

Jecht führte weiter aus, dass viele Typ-2-Diabetiker ihre Therapie als belastend und übermäßig kompliziert er- lebten. Daher seien einfache Behandlungsregime für die Adhärenz bei gleichzeitiger Verhinderung von Gewichtszunahme und Hypoglykämien so wichtig.

Dr. Stephan Arndt, Rostock, erörterte die derzeit verfügbaren oralen Erweiterungen der Metformin-Monotherapie. Derzeit stünden DPP-4-Hemmer, GLP-1-Analoga, Sulfonylharnstoff sowie SGLT-2-Hemmer zur Auswahl mit unterschiedlichen Anwendungsgebieten und Risiken. So erhöhten etwa Sulfonylharnstoffe das Hypoglykämierisiko und die Patienten nähmen zu, bei SGLT-2-Hemmern könne es zu Harnwegsinfektionen kommen. Ist das Ziel eine Gewichtsreduktion, seien GLP-1-Analoga im Vorteil. Aktuell seien DPP-4-Inhibitoren wie Sitagliptin Xelevia $^{\circledast}$, Januvia ${ }^{\circledR}$ und Saxagliptin $\left(\mathrm{Onglyza}^{\circledR}\right)$ als Standard anzusehen, von dem jedoch durchaus abgewichen werden könne.

\section{Sitagliptin bei Niereninsuffizienz uneingeschränkt einsetzbar}

Die besonderen Herausforderungen der oralen Diabetestherapie bei Patienten mit chronischer Niereninsuffizienz fasste Dr. Andreas Lueg vom Diabeteszentrum L1 in Hameln zusammen. 20\% aller Praxis-Patienten wiesen eine eGFR $<60 \mathrm{ml} /$ $\min / 1,73 \mathrm{~m}^{2}$ auf. Aufgrund der Nierenfunktionsstörung stünden für diese $\mathrm{Pa}$ tienten nur DPP-4-Hemmer zur Therapieerweiterung bzw. Monotherapie zur Verfügung. Nur Sitagliptin könne jedoch bis zur Dialyse angewendet werden. Saxagliptin sei ab einer eGFR von $15 \mathrm{ml} /$ $\min / 1,73 \mathrm{~m}^{2}$ kontraindiziert.

Jan Groh
- Fortbildungsveranstaltung "Stoffwechsel in Balance", Schwer-
punktthema:Der Typ-2-Diabetiker zwischen Hausarzt und Fach-
arzt; Berlin, Juni 2016 (Veranstalter:Berlin-Chemie)

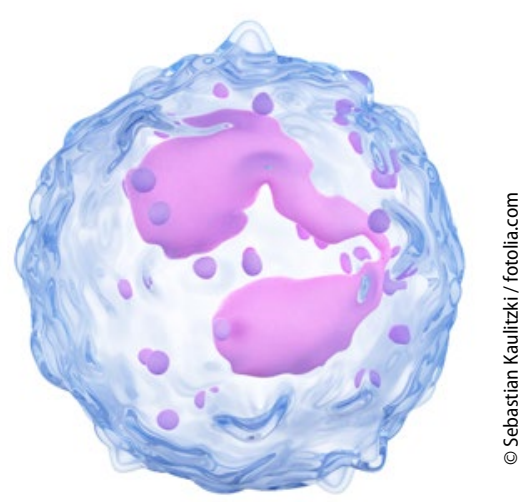
risiko sank ebenfalls signifikant. Die
Eosinophiler Granulozyt.

rol/Glykopyrronium (Ultibro Breezhaler ${ }^{\circledR}$ ) sind ICS/LABA-Kombinationen überlegen. In der ILLUMINATE-Studie mit Patienten ohne Exazerbationen verbesserte Indacaterol/Glycopyrronium die $\mathrm{FEV}_{1}$ um $100 \mathrm{ml}$ mehr als Salmeterol/Fluticason und senkte signifikant das Exazerbationsrisiko. In der LANTERN-Studie mit Patienten, die im Vorjahr eine Exazerbation erlitten hatten, stieg die $\mathrm{FEV}_{1}$ unter Indacaterol/Glycopyrronium um $75 \mathrm{ml}$ mehr als unter Salmeterol/Fluticason. Das Exazerbations-

Exazerbationen. 20\% der Patienten entwickeln unter ICS eine Pneumonie. Hinzu kommen langfristig erhöhte Risiken u. a. für Diabetes und Osteoporose.

\section{Eindeutige Studienlage}

Duale Bronchodilatatoren wie Indacate-

FLAME-Studie bestätigte, dass Indacaterol/Glycopyrronium $(1 \times 110 / 50 \mu \mathrm{g} / \mathrm{d})$ auch bei Patienten mit häufigen Exazerbationen Salmeterol/Fluticason $(2 \times$ $50 / 500 \mu \mathrm{g} / \mathrm{d}$ ) bezüglich des Schutzes vor Exazerbationen, der Lungenfunktion und der Lebensqualität überlegen ist. -

Dr. Dirk Einecke

- Satellitensymposium „Therapiefortschritt für symptomatische COPD- und Asthmapatienten", 122. Kongress der Deutschen Gesellschaft fürInnere Medizin; Mannheim, April 2016 (Veranstalter: Novartis)
Als letzte Leitlinien-gerechte Bastion
der inhalativen Kortikosteroide (ICS) gel-
ten Patienten mit chronisch obstruktiver
Lungenerkrankung (COPD) und häufi-
gen Exazerbationen. Nun zeigt die FLA-
ME-Studie, dass selbst bei diesen Patien-
ten eine doppelte Bronchodilatation mit
einem langwirksamen $\beta_{2}$-Agonisten
(LABA) und einem langwirksamen Mus-
karinantagonisten (LAMA) Indacaterol/
Glycopyrronium wirksamer das Exazer-
bationsrisiko senkt als die Kombination
aus Salmeterol/Fluticason - einem ICS
plus LABA. Es gibt aber eine Subgruppe
der COPD-Patienten mit häufigen Exa-
zerbationen, die ICS eventuell doch benö-
tigen könnten, erklärte Prof. Felix Herth,
Heidelberg. Das sind Patienten mit hoher
Bluteosinophilie.
ICS/LABA-Kombinationen schaden
jedoch COPD-Patienten ohne häufige 